\title{
Pharyngolaryngeal Pain, CTCAE
}

National Cancer Institute

\section{Source}

National Cancer Institute. Pharyngolaryngeal Pain, CT CAE. NCI Thesaurus. Code C143759.

A disorder characterized by a sensation of marked discomfort in the pharyngolaryngeal region. 